\title{
CHAOTIC MODE TRANSITION DYNAMICS IN HUMAN CIRCULATORY SYSTEMS
}

\author{
Taiki Narita* Noriyasu Homma ${ }^{* *}$ Masao Sakai ${ }^{* * *}$ \\ Makoto Yoshizawa ${ }^{* * * *}$ Kenichi Abe* \\ * Graduate School of Engineering, Tohoku University \\ Aoba, Aramaki, Aoba-ku, Sendai, 980-8579, JAPAN \\ ** Faculty of Medicine, Tohoku University \\ 2-1, Seiryo-machi, Aoba-ku, Sendai, 980-8575, JAPAN \\ *** Graduate School of Information Sciences, Tohoku \\ University, Kawauchi, Aoba-ku, Sendai, 980-8576, JAPAN \\ **** Information Synergy Center, Tohoku University \\ Aoba, Aramaki, Aoba-ku, Sendai, 980-8579, JAPAN
}

\begin{abstract}
In this paper, we propose a new chaotic itinerant model of mode transition dynamics in human circulatory systems. An essential emphasis of the model is on autonomous mode transition based on chaotic itinerancy of large-scale nonlinear systems. It is shown that the model possesses some important properties observed in the circulatory systems, such as mode transition and its controllability. Copyright (C)2005 IFAC
\end{abstract}

Keywords: Adaptive systems, Biomedical systems, Chaotic behavior, Modeling, Nonlinear Systems

\section{INTRODUCTION}

In human circulatory systems, behavior of time series of observable variables such as heart rate and blood pressure is changed depended on physiological conditions such as "sleep," "exercise," and "rest" (Narita et al., 2004b). The specific behaviors for different conditions can be referred to dynamical modes, and changes from specific modes into another may be regarded as mode transition. Thus, it might be useful to build a model that possesses an analyzable mode transition dynamics for revealing complex control mechanisms of human circulatory systems, in which the control mechanism may be adjusted according to the changes of specific conditions(Narita et al., 2004a).

A possible model of such mode transition may include specific functions of dynamics that are potentially required for different modes. Then we just select a function among those candidates corresponding to the specific mode. It is expected, however, that such switching mechanism that follows mode transition can be very complicated for controlling human circulatory systems as largescale nonlinear systems.

On the other hand, chaotic itinerancy(Kaneko, 1990) is one of plausible models of such complex mode transition. The chaotic itinerancy can be observed in nonlinear systems with large degree of freedom and its behavior is more complex than that of chaotic one with relatively small degree of freedom. An advantage of the chaotic itinerancy model is in its simple rule to generate such complex dynamics. Fortunately, even the behavior is defined by a simple rule, chaotic itinerancy possesses an autonomous and complex mode transition dynamics intrinsically. 
In this paper, we propose a novel chaotic itinerant model to analyze such complex mode transition dynamics in human circulatory systems. Then we develop a control mechanism of the autonomous mode transition of the proposed model by using external inputs that can be generated by the autonomous nerve system in the circulatory systems. Simulation results demonstrate usefulness and plausibility of the proposed chaotic itinerant model.

\section{CHAOTIC ITINERANT MODEL}

Chaotic itinerancy can be defined as complex dynamics of such a system in which (i) state variables of the system stay in one of pseudo stable modes, or pseudo and sub-attractors for a while, and then (ii) transit to the other mode autonomously through chaotic complex orbits. Chaotic itinerancy can be observed in many nonlinear systems with large degree of parameter freedom(Kaneko, 1990; Tsuda, 1991; Ikeda et $a l ., 1989)$. In this section, we will describe chaotic itinerant behavior briefly by using a globally coupled map first, and then the new model will be proposed to control the autonomous mode transition of complex physiological dynamics.

\subsection{Globally coupled map}

Globally coupled map (GCM)(Kaneko, 1990) is defined as

$$
s_{i}(t+1)=(1-\varepsilon) g\left(s_{i}(t)\right)+\frac{\varepsilon}{N} \sum_{j=1}^{N} g\left(s_{j}(t)\right)(1)
$$

where $s_{i}, i=1,2, \ldots, N$, are outputs of mapping units, $N$ is the number of units, $t=0,1,2, \ldots$ denote discrete time, $\varepsilon, 0 \leq \varepsilon \leq 1$, is a positive constant, and $g($.$) is a chaotic mapping function,$ respectively.

The first term of the right-hand side in Eqn. (1) implies asynchronous behavior because of the chaotic function, while the second term of the right-hand side synchronizes the states by the mean field. Thus, a wide range of dynamical behavior from synchronous to asynchronous or chaotic one can be observed by changing the parameter value $\varepsilon$ of the GCM system. Let us introduce a notion of "cluster" implying a set of states that are synchronized with each other, and the number of clusters in the GCM at time $t, C(t)$. Note that the number of clusters represents the dynamic mode of the GCM. We can observe chaotic itinerancy in the time series of the number of clusters $C(t)$ if the parameter $\varepsilon$ is appropriate for the mapping function $g$ (see
Fig. 1). That is, the system stays in one of pseudo stable modes for a duration, where the number of clusters $C(t)$ is stable or a constant, and then transit autonomously to another mode through chaotic complex orbits in which almost all the units behave asynchronously and the number of clusters $C(t)$ is the number of units $N$ approximately.

\subsection{Proposed model}

Chaotic itinerancy implies autonomous mode transition, but if it is completely "autonomous" or out of control without respect to the physiological conditions, it might not be desirable for a model of human circulatory systems. In this paper, we propose a novel model that not only involves autonomous mode transition of chaotic itinerancy, but also can control the mode transition by using external inputs representing physiological conditions.

Let us use the following logistic map with a parameter $a$ and the external input as the chaotic function $g($.$) in Eqn. (1)$

$$
g(s)=1-(a-I(t)) s^{2} \equiv 1-a^{\prime} s^{2}
$$

where $a^{\prime}=a-I(t)$. Dynamic complexity of the function is depended on the parameter $a$ '(Kaneko and Tsuda, 1996). Thus, we can control the asynchronous or chaotic contribution to the GCM by changing the external input $I(t)$. For example, if we choose $a=1.90$, a large input makes the system be in a synchronous mode where the number of cluster is steady and small. In the following, autonomous model behavior without the external inputs and controlled behavior by using external inputs will be compared first, then the mode transition control mechanism will be developed by using the external control inputs.

2.2.1. Autonomous behavior Let us consider an autonomous behavior of the system with no external input $I(t)=0$. Fig. 1 shows the number of clusters $C(t)$, as a function of time $t$, of the system with $N=10, a=1.90$, and $\varepsilon=0.186$. Note that chaotic itinerancy in the time variation of the number of clusters can be observed. That is, the number of clusters is steady for a short period, but at the same time it is unstable for a long-term.

\subsubsection{Controlled behavior with external inputs}

Fig. 3 shows the number of clusters $C(t)$, as a function of time $t$, of a system controlled by the external input $I(t)$ as shown in Fig. 2. The other conditions are the same as in Section 2.2.1. The result implies that the number of clusters can be 


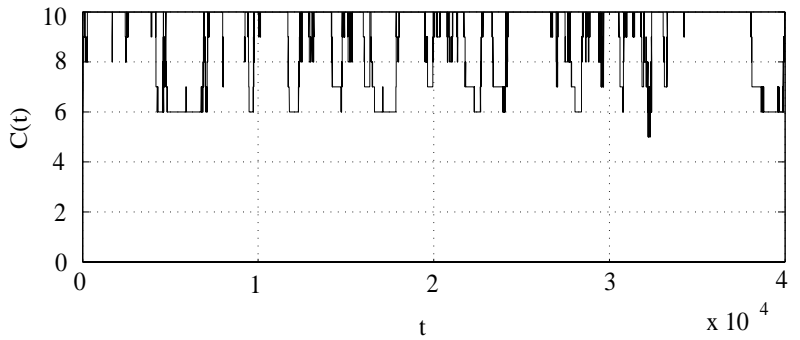

Fig. 1. Autonomous behavior without external input $I(t)=0$. Chaotic itinerancy can be observed in time variation of the number of clusters of the system with $N=10, a=1.90$, and $\varepsilon=0.186$.

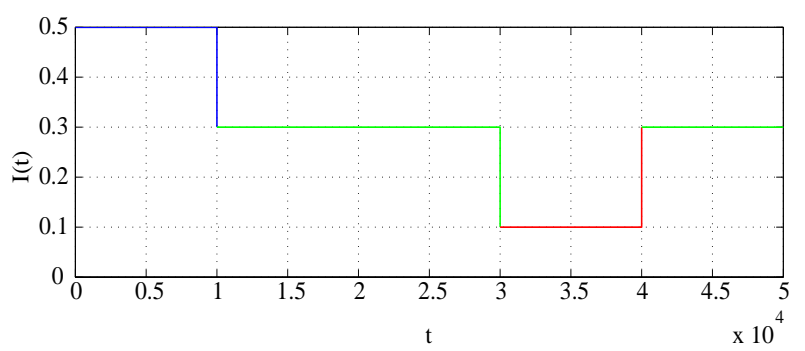

Fig. 2. The external input $I(t)$ as a function of time $t$.

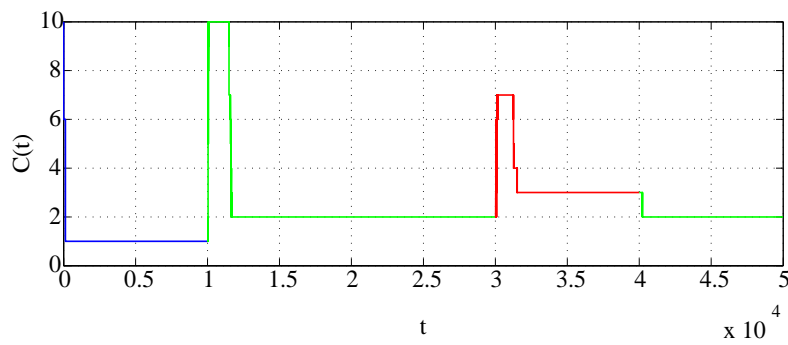

Fig. 3. Controlled behavior by the external input $I(t) \neq 0$ shown in Fig. 2. Controlled mode transition can be observed in time variation of the number of clusters of the system with $N=10, a=1.90$, and $\varepsilon=0.186$.

stable for a steady input value and changed from a steady value to another according to the changes of the inputs through the chaotic or asynchronous mode, in which the number of clusters $C(t)=N$ approximately. In other words, the autonomous mode transition of the GCM system in Eqn. (1) can be controlled by using the external input.

2.2.3. Mode transition control mechanism In general, it is almost impossible to represent all the time series of any dynamics by using only a GCM system defined as Eqns. (1) and (2). Thus we need another model to represent a desired time series of target systems in which chaotic itinerancy can be observed. The proposed idea for implementing a desired time series of the chaotic itinerant system is to change the dynamic mode of

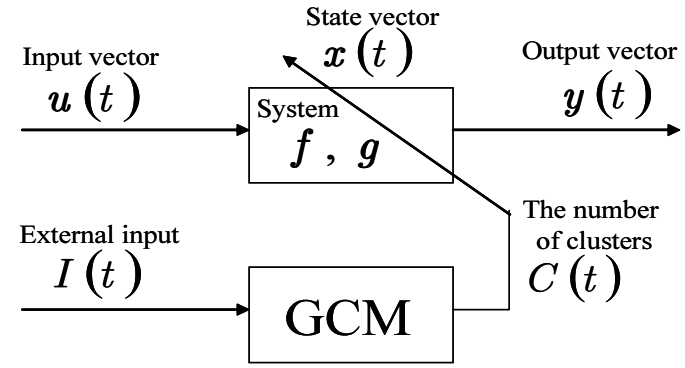

Fig. 4. The block diagram of the proposed mode transition control mechanism.

the target system autonomously by using complex mode transition of the GCM in Eqns. (1) and (2). In other words, we separate the representing systems $\boldsymbol{f}$ and $\boldsymbol{g}$ of the target time series from a mode transition mechanism of chaotic itinerancy. The block diagram of the mode transition control mechanism proposed in this paper is depicted in Fig. 4. Let us consider an $n$ dimensional system given as

$\left\{\begin{aligned} \boldsymbol{x}(t+1) & =\boldsymbol{f}(\boldsymbol{x}(t), \boldsymbol{u}(t)) \\ \boldsymbol{y}(t) & =\boldsymbol{g}(\boldsymbol{x}(t), \boldsymbol{u}(t))\end{aligned}\right.$

where

$$
\begin{aligned}
\boldsymbol{x}(t) & =\left[\begin{array}{llll}
x_{1}(t) & x_{2}(t) & \ldots & x_{n}(t)
\end{array}\right]^{T} \in \Re^{n} \\
\boldsymbol{u}(t) & =\left[\begin{array}{llll}
u_{1}(t) & u_{2}(t) & \ldots & u_{r}(t)
\end{array}\right]^{T} \in \Re^{r} \\
\boldsymbol{y}(t) & =\left[\begin{array}{llll}
y_{1}(t) & y_{2}(t) & \ldots & y_{m}(t)
\end{array}\right]^{T} \in \Re^{m} \\
\boldsymbol{f}(\boldsymbol{x}, \boldsymbol{u}) & =\left[\begin{array}{lllll}
f_{1}(\boldsymbol{x}, \boldsymbol{u}) & f_{2}(\boldsymbol{x}, \boldsymbol{u}) & \ldots & f_{n}(\boldsymbol{x}, \boldsymbol{u}
\end{array}\right]^{T} \in \Re^{n} \\
\boldsymbol{g}(\boldsymbol{x}, \boldsymbol{u}) & =\left[\begin{array}{lllll}
g_{1}(\boldsymbol{x}, \boldsymbol{u}) & g_{2}(\boldsymbol{x}, \boldsymbol{u}) & \ldots & g_{m}(\boldsymbol{x}, \boldsymbol{u}
\end{array}\right]^{T} \in \Re^{m}
\end{aligned}
$$

Here $\boldsymbol{x}$ denotes the state vector, $\boldsymbol{u}$ denotes the input vector, $\boldsymbol{y}$ denotes the output vector, and $f_{p}, g_{q}, p=1,2, \cdots, n, q=1,2, \cdots, m$, are nonlinear functions. Let us control the parameter of the system by using the number of clusters of the GCM given in Eqns. (1) and (2). Suppose that $f_{p}$ in Eqn. (7) is given as a linear formation

$$
\begin{aligned}
f_{p}(\boldsymbol{x}, \boldsymbol{u})= & a_{p_{1}} x_{1}+a_{p_{2}} x_{2}+\cdots+a_{p_{n}} x_{n} \\
& +b_{p_{1}} u_{1}+b_{p_{2}} u_{2}+\cdots+b_{p_{r}} u_{r}
\end{aligned}
$$

System functions $f_{p}^{j}, j=1,2, \ldots, m$ corresponding to specific modes of the number of clusters can then be given as

$$
\begin{aligned}
f_{p}^{j}(\boldsymbol{x}, \boldsymbol{u})= & a_{p_{1}}^{j} x_{1}+a_{p_{2}}^{j} x_{2}+\cdots+a_{p_{n}}^{j} x_{n} \\
& +b_{p_{1}}^{j} u_{1}+b_{p_{2}}^{j} u_{2}+\cdots+b_{p_{r}}^{j} u_{r}
\end{aligned}
$$

Here if there are functions $h_{p_{k}}^{j}, H_{p_{l}}^{j}$, of the number of clusters $C_{j}$ corresponding to mode $j$ such that

$$
\begin{aligned}
a_{p_{k}}^{j} & =h_{p_{k}}^{j}\left(C_{j}\right), \quad k \in\{1,2, \ldots, n\} \\
b_{p_{l}}^{j} & =H_{p_{l}}^{j}\left(C_{j}\right), \quad l \in\{1,2, \ldots, r\}
\end{aligned}
$$


then any time series that can be generated by such system $f_{i}$ can be formulated as a controllable dynamics by using the proposed model with external inputs. Much the same is true on $\boldsymbol{g}$. We will demonstrate this controllable chaotic itinerant behavior in the following simulation studies.

\section{SIMULATION STUDIES}

\subsection{Mode transition control for Rössler system}

As a target system of Eqn. (9) in continuous time domain, we consider the following Rössler system given as

$$
\begin{aligned}
& d x / d t=-y-z \\
& d y / d t=x+a y \\
& d z / d t=b+z(x-c)
\end{aligned}
$$

Let us define the parameters of the system by using the notation in Eqn. (11) as

$$
\begin{aligned}
& a=0.36 \\
& b=0.4 \\
& c=C(t)
\end{aligned}
$$

where $C(t)$ is the number of clusters of the GCM. In this simulation, we used the same changes of external inputs and the corresponding number of clusters $C(t)$ shown in Figs 2 and 3, respectively. Figs. 5 and 6 show the attractor of the controlled Rössler system and its time series of one of the states, $x(t)$. To measure time variation of the dynamical complexity, we calculated the largest Lyapunov exponent $\lambda$ (Yano et al., 2002) that can be given as an average exponential expanding rate of the maximum direction of the orbit in the state space. The largest Lyapunov exponent $\lambda$ is 0.031 for duration $0 \leq t<10000$ where the number of cluster $C(t)=1$, representing mode 1. $\lambda=0.049$ for $10000 \leq t<30000$ and for $40000 \leq t<50000$ where $C(t)=2$, representing mode 2 , and $\lambda=0.107$ for $30000 \leq t<40000$ where $C(t)=3$, representing mode 3 , respectively. We can see that modes of time series and the largest Lyapunov exponent follow the source mode transition represented by the changes of the number of clusters of the GCM.

As is clear from the result, the proposed model can generate a chaotic time series of Rössler system, and its intrinsic autonomous mode transition can be controlled by using external inputs. Accordingly, if we use another system instead of Rössler system considered in this example, we can develop such autonomous and controlled behavior for any target systems as well. In the next example, in order to apply the proposed control mechanism to a practical problem, a model of human circulatory systems will be considered.

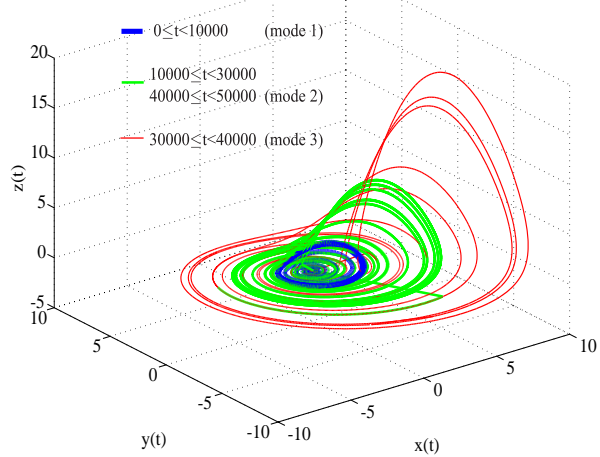

Fig. 5. Attractor of the controlled Rössler system.

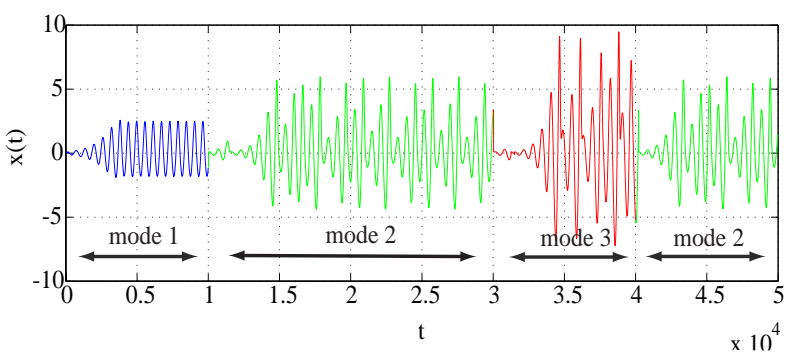

Fig. 6. Time series of the $x(t)$ of the controlled Rössler system.

3.2 Mode transition control for a model of human circulatory system

Let us consider a Windkessel model as a model of human circulatory systems (Cavalcanti and Belardinelli, 1996). The block diagram of the model with three elements is illustrated in Fig. 7.

The three elements of the model are a peripheral resistance $R$, an arterial compliance $C_{a}$, and an aorta characteristic impedance $r$. In this model, the circulatory system can be represented by an electrical circuit model of which input is aortic flow $Q$ and output is aortic pressure $P$. Then, the three-element Windkessel model can be given as

$$
\begin{aligned}
\frac{d P_{s}(t)}{d t} & =-\frac{1}{R C_{a}} P_{s}(t)+\frac{1}{C_{a}} Q(t) \\
P(t) & =P_{s}(t)+r Q(t)
\end{aligned}
$$

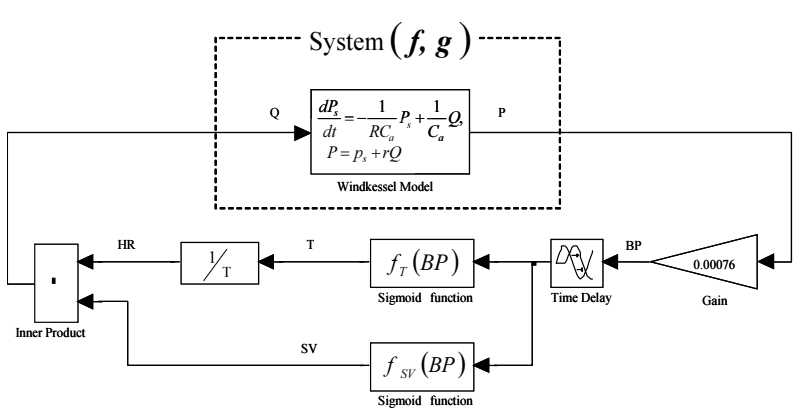

Fig. 7. The block diagram of the three-element Windkessel model. 
where $P_{s}$ denotes a state variable of the system (Cavalcanti and Belardinelli, 1996). As shown in Fig. 7, cardiac cycle $T$ and systolic output $S V$ are, respectively, given as sigmoid functions of arterial pressure $B P$

$$
\begin{gathered}
T=f_{T}(B P)=T_{s}+\frac{T_{m}-T_{s}}{1+\gamma e^{-\alpha \frac{B P}{P_{n}}}} \\
S V=f_{S V}(B P)=\frac{S V_{\max }}{1+\beta\left(\frac{B P}{P_{v}}-1\right)^{-k}}
\end{gathered}
$$

where

$$
\begin{aligned}
T_{s} & =0.66[\mathrm{~s}] \\
T_{m} & =1.2[\mathrm{~s}] \\
P_{n} & =89[\mathrm{mmHg}] \\
\alpha & =31 \\
\gamma & =6.7 \times 10^{13} \\
S V_{\text {max }} & =86\left[\mathrm{~cm}^{3}\right] \\
P_{v} & =25[\mathrm{mmHg}] \\
\beta & =72 \\
k & =7
\end{aligned}
$$

The constant values given above are physiologically plausible(Cavalcanti and Belardinelli, 1996). In this case, we can make graphs of these functions $f_{T}$ and $f_{S V}$ as shown in Figs. 8 and 9, respectively.

Let us define the system parameters followed by the notation given in Eqn. (11) as

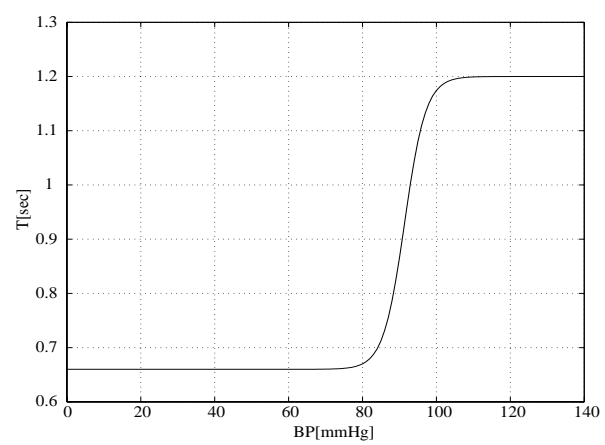

Fig. 8. Cardiac cycle $T$ as a sigmoid function of arterial pressure $B P$.

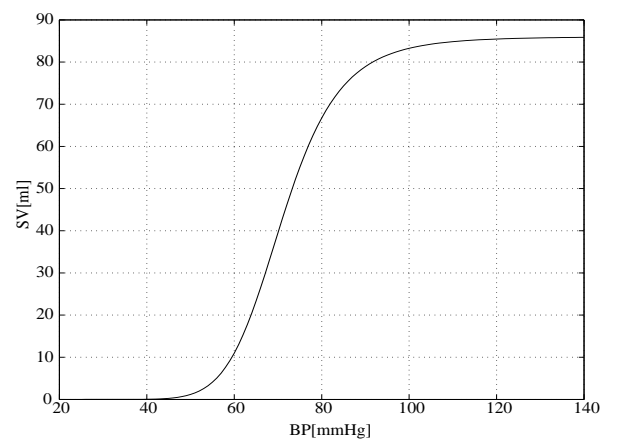

Fig. 9. Systolic output $S V$ as a sigmoid function of arterial pressure $B P$.

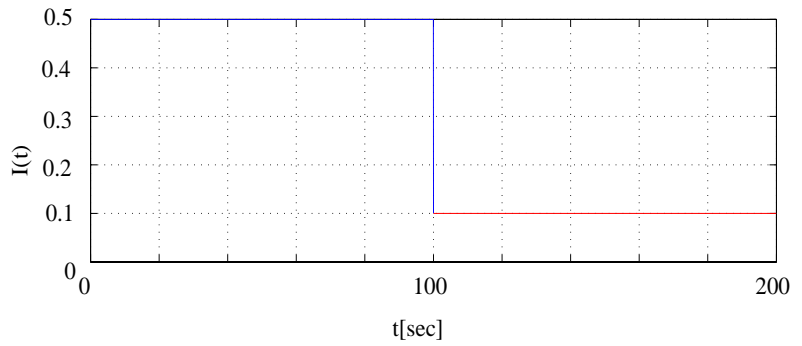

Fig. 10. The external input $I(t)$ as a function of time $t$.

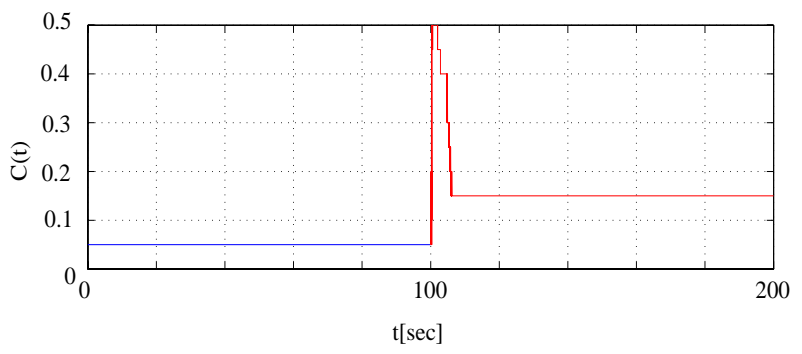

Fig. 11. Time variation of the number of clusters of the system with $N=10, a=1.90$, and $\varepsilon=0.186$, controlled by the external input shown in Fig. 10.

$$
\begin{aligned}
R & =1160+120 / C(t)\left[\mathrm{dyns} / \mathrm{cm}^{5}\right] \\
C_{a} & =1 \times 10^{-3}\left[\mathrm{~cm}^{5} / \mathrm{dyn}\right] \\
r & =52\left[\mathrm{dyns} / \mathrm{cm}^{5}\right]
\end{aligned}
$$

In this simulation, we used the changes of external inputs $I(t)$ and the corresponding number of clusters $C(t)$ shown in Figs. 10 and 11, respectively. Anterior half of external inputs $I(t)$ in Fig. 10 is corresponding to human rest state, and posterior half of external inputs $I(t)$ in Fig. 10 is corresponding to human load state. Also, the time delay in Fig. 7 was set to 2.5. Fig. 12 shows the attractor of the controlled Windkessel model. Arterial pressure $B P$, heart rate $H R$, and systolic output $S V$ as functions of time $t$ are, respectively, shown in Figs. 13, 14, and 15. The largest Lyapunov exponent $\lambda$ is 3.7206 for duration $0 \leq t<100$ where $C(t)=1$, representing mode $1 . \lambda=6.8375$ for duration $100 \leq t<200$ where $C(t)=3$, representing mode 2 . We can see that the orbit in the attractor suggests that existence of the controlled mode transition. The trajectories of mode 1 for $0 \leq t<100$ and mode 2 for $100 \leq t<200$ are shown as bold and thin lines in Fig. 12, respectively. Accordingly, the controlled mode transitions can be observed in the time series of states $B P, H R, S V$, and the dynamical complexity $\lambda(t)$. The transition of the largest Lyapunov exponent from 3.7206 (mode 1) to 6.8375 (mode 2) implies that behavior in mode 1 is more periodic or simple than that of mode 2 . The periodicity can be observed in the anterior 
part of the time series of $\mathrm{BP}, \mathrm{HR}$, and $\mathrm{SV}$ as shown in Figs. 13, 14, and 15, respectively. That is, the time series are more complex after the mode transition at $t=100$.

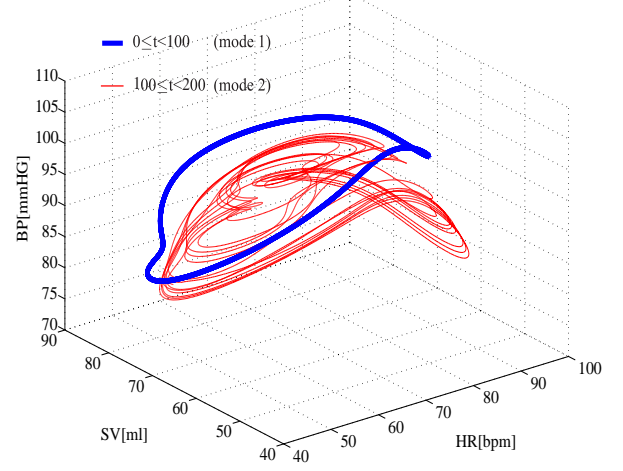

Fig. 12. Attractor of the controlled Windkessel model.

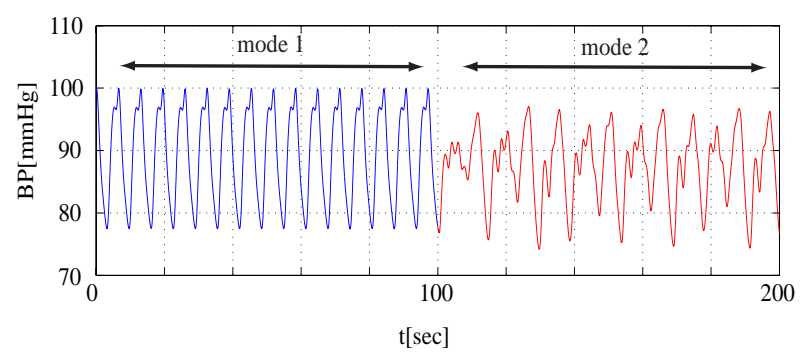

Fig. 13. Time series of the arterial pressure $B P(t)$ of the controlled Windkessel model.

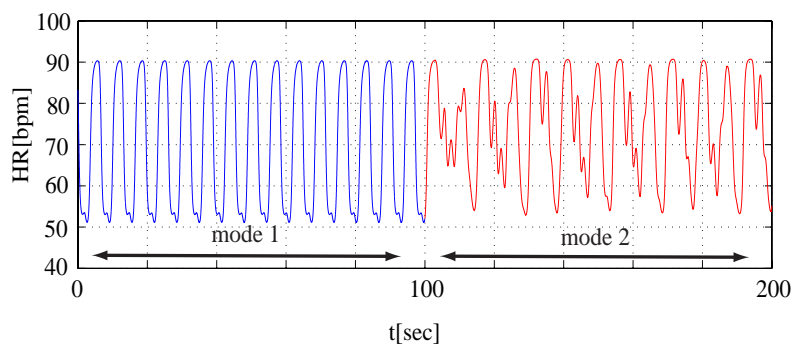

Fig. 14. Time series of the heart rate $H R(t)$ of the controlled Windkessel model.

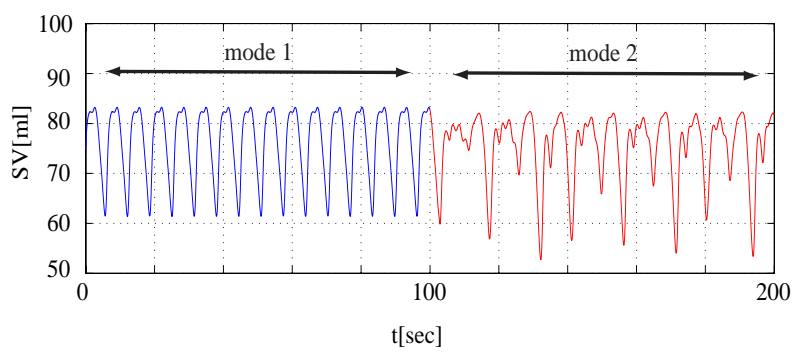

Fig. 15. Time series of the systolic output $S V(t)$ of the controlled Windkessel model.

\section{CONCLUSIONS}

In this paper, we have developed a new chaotic itinerant model for mode transitions control. Simulation results demonstrate that the intrinsic autonomous mode transition of some target systems can be controlled by external inputs.

Since there is no model that possesses such complex mode transition explicitly, the goal of most of conventional control systems applied to human circulatory systems is to keep the system being steady in a fixed mode such as a rest condition.

On the other hand, the proposed model possesses a controllable autonomous mode transition intrinsically. Note that the autonomous behavior is unique and completely different from the other models. It might be expected that we can develop a control system that is not only to keep a steady state, but also to apply to such complex mode transition. To achieve this, relations between state variables of the circulatory systems and the dynamical modes are needed to be investigated more clearly.

\section{REFERENCES}

Cavalcanti, S. and E. Belardinelli (1996). Modeling of cardiovascular variability using a differential delay equation. IEEE Trans. Biomed. Eng. 43(10), 982-989.

Ikeda, K., K. Otsuka and K. Matsumoto (1989). Maxwell bloch turbulence. Prog. Theor. Phys. (Supplement) 99, 295-324.

Kaneko, K. (1990). Clustering, coding, switching, hierarchical ordering, and control in network of chaotic elements. Physica 41 D, 137-172.

Kaneko, K. and I. Tsuda (1996). Chaotic scenario of complex systems. Asakura-syoten. Tokyo (in Japanese).

Narita, T., N. Homma, M. Sakai, M. Yoshizawa and K. Abe (2004a). Chaotic itinerancy model for human circulatory systems dynamics. Proc. SICE Annual Conference 2004 pp. 2124-2129.

Narita, T., N. Homma, M. Sakai, M. Yoshizawa and K. Abe (2004b). Chaotic itinerant model of mode transition dynamics. Bull. Sch. Health Sci. Tohoku Univ. 13(2), 101-112 (in Japanese).

Tsuda, I. (1991). Chaotic itinerancy as a dynamical basis of hermeneutics in brain and mind, world futures. World Futures 31, 105-122.

Yano, M., N. Homma, M. Sakai and K. Abe (2002). Phase-space reconstruction from observed time series using lyapunov spectrum analysis. Proc. SICE Annual Conference 2002 pp. 726-731. 